\title{
Imaging Cavernous Malformations
}

Cavernous malformations (CMs) represent $\sim 10 \%$ to $15 \%$ of vascular malformations. Their incidence is $\sim 0.5 \%$ at autopsy. They have low pressure, slow flow, and tend to be angiographically occult (Tomlinson et al., 1994). They are commonly referred to as cavernous angiomas or cavernomas. CMs consist of enlarged sinusoidal vascular spaces that have thin walls devoid of smooth muscle and normal endothelium. These form compact masses within central nervous system (CNS) parenchyma or associated structures without normal interspersed tissue. The thin walls lack normal endothelium and are prone to leakage of blood elements. Deposition of blood products in and around CMs may be extensive (Glendhill et al., 2000).

\section{CAVERNOUS MALFORMATION}

BASIC

The following sequences comprise our basic protocol for evaluating cavernous malformations:

1. Three-plane positioning scout.

2. Noncontrast $T_{1}$-weighted transverse.

3. Transverse gradient echo.

4. Noncontrast coronal proton density (PD)/ $/ T_{2}$-weighted turbo spin echo.

5. Post contrast $T_{1}$-weighted transverse.

And an optional:

6. Post contrast $T_{1}$-weighted transverse with flow compensation.

Although the actual scan time is $12 \mathrm{~min}$ and $19 \mathrm{sec}$ (or $15 \mathrm{~min}$ and $46 \mathrm{sec}$ with the optional sequence), because of setup time between sequences and the time necessary to inject the gadolinium, we tell the patient that this exam will take $\sim 30 \mathrm{~min}$. Except for the three-plane positioning scout, if the patient just had a "routine" cranial MR exam, we perform only those sequences necessary to complete this protocol. Table A2.2.1 lists the necessary equipment needed to perform the imaging sequences in this unit.

NOTE: Be sure that technologists and nurses have immediate access to any emergency equipment that may be relevant to a given study, or that may be needed for a particular patient, such as a crash cart or oxygen.

\begin{tabular}{ll}
$\begin{array}{l}\text { Table A2.2.1 Equipment Specifications Needed to Perform the } \\
\text { Following Imaging Sequences }\end{array}$ \\
$\begin{array}{ll}\text { Coil type } & \text { Circularly polarized head coil } \\
\text { Field strength } & 1.5 \mathrm{~T} \\
\text { Gradient strength } & \text { Minimum of } 15 \mathrm{mT} / \mathrm{m} \text { (or whatever the } \\
& \text { system permits) } \\
\text { Positioning cushion or } & \text { Yes } \\
\text { head stabilizers } & \text { Yes } \\
\text { Knee cushion } & \text { Yes } \\
\text { Use of contrast agents } & \text { If patient requires sedation } \\
\text { Pulse oximeter } & \end{array}$ \\
\hline
\end{tabular}




\section{Materials}

Normal saline $(0.9 \% \mathrm{NaCl})$

Sterile extravascular contrast agent (e.g., Magnevist, Omniscan, Prohance)

\section{Set up patient and equipment}

1. Interview the patient to assess for contraindications such as cardiac pacemaker, implanted mechanical devices, and/or ferromagnetic materials. Also, determine if the patient will need sedation medication, which will necessitate the need for appropriate monitoring equipment.

Each patient or legal guardian prior to bringing the patient into the exam area signs a screening form.

The presence of ferromagnetic materials may be a health hazard to the patient while inside the magnet and/or adversely affect image quality. To determine the safety of scanning such ferromagnetic materials see Shellock (1996).

The presence of ferromagnetic materials in the globe of the eye is contraindicated for MRI. Patients with prior metal exposure to the eye should have plain $x$-rays of the orbital area to ensure that all metal has been removed prior to placing them in the magnetic field.

2. If the procedure is a research protocol, have the patient sign any necessary consent form.

3. Request the patient to change into a gown and remove all personal effects, such as jewelry, hearing aids, glasses, etc., prior to entering the MRI scan room. All personal belongings should be secured during the examination.

4. Have the patient wash off any mascara and other makeup to avoid local tissue heating and artifacts.

5. Explain the procedure to the patient and record relevant clinical history. Ensure that the patient understands what is expected and ask them if they have had questions answered appropriately.

6. Set up the exam room by securing the circularly polarized (CP)-head coil onto the table and providing a clean exam table.

7. Escort the patient to the MR examination room and position them according to the exam to be performed. Review the following items with the patient:

a. Provide earplugs or headphones to the patient to minimize the gradient noise but assure them that they will still be able to hear you.

b. Provide the patient with a safety squeeze-bulb and demonstrate how it works. Explain to the patient that they may use the squeeze-bulb if they need assistance during the exam.

c. Explain to the patient that you will be talking to them between imaging sequences (i.e., when the loud knocking noise stops).

d. Explain to the patient that it is imperative that they remain motionless during the loud knocking noise to ensure good results. Also, explain that they should not reposition their body between imaging sequences.

e The patient may call out at any time if he or she feels it necessary.

f. Provide the patient with an estimate of the examination length. If this entire protocol will be followed, we suggest that the exam will take approximately half an hour.

8. Position a support under the patient's knees to enhance comfort.

Imaging Cavernous

Malformations

A2.2.2

Supplement 5 
11. Use the laser light to center on the patient's nasion.

12. Advance the patient couch to isocenter of the magnet.

Once this step has been performed, so long as the patient does not move on the table, the table itself can be moved and then replaced in the same position as before without jeopardizing the positioning of one scan relative to another.

\section{Sequence 1: Rapid three-plane positioning scout}

13. To validate the patient's position and to have a reference to prescribe successive imaging sequences, acquire a three-plane orthogonal scout sequence. See Table A2.2.2 for specific parameters.

Most MR scanners can be programmed to acquire the scout automatically after coil tuning or after the patient has been placed in isocenter (for systems that do not require tuning). Siemens acquires the scout in three planes by selecting "various".

\section{Sequence 2: Noncontrast $T_{1}$-weighted transverse}

14. Run a noncontrast $T_{1}$-weighted transverse spin echo sequence according to Table A2.2.3.

On this study we will be looking for multiple round areas of high $T_{1}$-weighted signal, which are usually seen in the central portion of these lesions. These deposits often demonstrate a "popcorn" appearance that is characteristic of many of these lesions.

\section{Sequence 3: Transverse gradient echo}

15. Display both the sagittal and transverse scout images in two separate quadrants on the scan monitor. Change imaging parameters to those listed in Table A2.2.4. Position slices to the midline of the sagittal scout image. Adjust position of field of view (FOV) off transverse scout image.

16. Instruct the patient to remain motionless as the scan will begin and last for $\sim 5 \mathrm{~min}$.

17. Run a transverse gradient echo according to Table A2.2.4.

Table A2.2.2 Imaging Parameters for Sequence 1 (Scout Sequence)

\begin{tabular}{ll}
\hline Patient position & $\begin{array}{l}\text { Supine } \\
\text { Gcan type }\end{array}$ \\
Imaging planes (orientation) & $\begin{array}{l}\text { Transverse, sagittal, and coronal } \\
\text { (various) }\end{array}$ \\
Central slice or volume center & Nasion \\
Echo time $\left(T_{\mathrm{E}}\right)$ & $6 \mathrm{msec}$ \\
Repeat time $\left(T_{\mathrm{R}}\right)$ & $15 \mathrm{msec}$ \\
Flip angle $(\mathrm{FA})$ & $30^{\circ}$ \\
Fields of view $\left(\mathrm{FOV}_{\mathrm{x}}, \mathrm{FOV}_{\mathrm{y}}\right)$ & $300 \mathrm{~mm}, 300 \mathrm{~mm}$ \\
Resolution $(\Delta x, \Delta y)$ & $1.17 \mathrm{~mm}, 2.34 \mathrm{~mm}$ \\
Number of data points collected $\left(N_{\mathrm{x}}, N_{\mathrm{y}}\right)$ & 256,128 \\
Slice thickness $(\Delta z)$ & $8 \mathrm{~mm}$ \\
Number of slices & 3 (one per orientation) \\
Slice gap & Not applicable \\
Number of acquisitions $\left(N_{\mathrm{acq}}\right)$ & 1 \\
Swap read and phase encoding & No \\
Slice location & Not applicable \\
Saturation pulses & Not applicable \\
Slice series & Ascending (caudal to cranial) \\
Scan time & 7 sec \\
\hline
\end{tabular}

Cerebral Venous Lesions

A2.2.3 
Table A2.2.3 Imaging Parameters for Sequence 2 (Transverse $T_{1}$-Weighted Spin Echo)

\begin{tabular}{ll}
\hline Patient position & Supine \\
Scan type & Spin echo \\
Imaging plane (orientation) & Transverse \\
Central slice or volume center & Slices centered to midbrain on sagittal \\
& scout image \\
Echo time $\left(T_{\mathrm{E}}\right)$ & $15 \mathrm{msec}$ \\
Repeat time $\left(T_{\mathrm{R}}\right)$ & $560 \mathrm{msec}$ \\
Flip angle $(\mathrm{FA})$ & $90^{\circ}$ \\
Fields of view $\left(\mathrm{FOV}_{\mathrm{x}}, \mathrm{FOV}_{\mathrm{y}}\right)$ & $230 \mathrm{~mm}, 230 \mathrm{rmm}$, with $r=3 / 4$ \\
& $($ rectangular field of view $)$ \\
Resolution $(\Delta x, \Delta y)$ & $0.90 \mathrm{~mm}, 0.90 \mathrm{~mm}$ \\
Number of data points collected $\left(N_{\mathrm{x}}, N_{\mathrm{y}}\right)$ & $256,256 r$, with $r=3 / 4($ rectangular \\
& field of view) \\
Slice thickness $(\Delta z)$ & $6 \mathrm{~mm}$ \\
Number of slices & 20 \\
Slice gap & $1.8 \mathrm{~mm}(-30 \%)$ \\
Number of acquisition $\left(N_{\mathrm{acq}}\right)$ & 2 \\
Read direction & Anterior-posterior \\
Slice location & Vertex to skull base \\
Saturation pulses & None \\
Slice series & Interleaved \\
Scan time & 3 min, 38 sec \\
\hline
\end{tabular}

Table A2.2.4 Imaging Parameters for Sequence 3 (Transverse Gradient Echo)

Patient position

Scan type

Imaging plane (orientation)

Central slice or volume center

Echo time $\left(T_{\mathrm{E}}\right)$

Repeat time $\left(T_{\mathrm{R}}\right)$

Flip angle (FA)

Fields of view $\left(\mathrm{FOV}_{\mathrm{x}}, \mathrm{FOV}_{\mathrm{y}}\right)$

Resolution $(\Delta x, \Delta y)$

Number of data points collected $\left(N_{\mathrm{x}}, N_{\mathrm{y}}\right)$

Slice thickness $(\Delta z)$

Number of slices

Slice gap

Number of acquisition $\left(N_{\mathrm{acq}}\right)$

Swap read and phase encoding

Read direction

Slice locations

Saturation pulses

Slice series

Scan time
Supine

2-D Gradient echo

Transverse

Slices centered to midline on sagittal scout image

$26 \mathrm{msec}$

$750 \mathrm{msec}$

$30^{\circ}$

$230 \mathrm{~mm}, 230 r \mathrm{~mm}$, with $r=3 / 4$ (rectangular field of view)

$0.90 \mathrm{~mm}, 0.90 \mathrm{~mm}$

256, 256r, with $r=3 / 4$ (rectangular field of view)

$5 \mathrm{~mm}$

18

$1.5 \mathrm{~mm}(30 \%)$

2

No

Posterior-anterior

Vertex to base of skull

No

Interleaved

$4 \mathrm{~min}, 50 \mathrm{sec}$ 
Table A2.2.5 Imaging Parameters for Sequence 4: Coronal Proton Density/ $T_{2}$-Weighted Turbo Spin Echo Images

\begin{tabular}{|c|c|}
\hline Patient position & Supine \\
\hline Scan type & Turbo spin echo (dual) \\
\hline Imaging plane (orientation) & Coronal \\
\hline Central slice or volume center & $\begin{array}{l}\text { Slices centered to midbrain on sagittal } \\
\text { scout image }\end{array}$ \\
\hline Echo time $\left(T_{\mathrm{E}}\right)$ & $15 \mathrm{msec}$ and $105 \mathrm{msec}$ \\
\hline Echo train length (ETL) & 7 \\
\hline Repeat time $\left(T_{\mathrm{R}}\right)$ & $4500 \mathrm{msec}$ \\
\hline Flip angle (FA) & $180^{\circ a}$ \\
\hline Fields of view $\left(\mathrm{FOV}_{\mathrm{x}}, \mathrm{FOV}_{\mathrm{y}}\right)$ & $\begin{array}{l}230 \mathrm{~mm}, 230 r \mathrm{~mm} \text {, with } r=3 / 4 \\
\text { (rectangular field of view) }\end{array}$ \\
\hline Resolution $(\Delta x, \Delta y)$ & $0.90 \mathrm{~mm}, 0.90 \mathrm{~mm}$ \\
\hline Number of data points collected $\left(N_{\mathrm{x}}, N_{\mathrm{y}}\right)$ & $\begin{array}{l}256,256 r \text {, with } r=3 / 4 \text { (rectangular } \\
\text { field of view) }\end{array}$ \\
\hline Slice thickness $(\Delta z)$ & $5 \mathrm{~mm}$ \\
\hline Number of slices & 24 \\
\hline Slice gap & $1.5 \mathrm{~mm}$ \\
\hline Number of acquisition $\left(N_{\mathrm{acq}}\right)$ & 1 \\
\hline Swap read and phase encoding & No \\
\hline Slice location & $\begin{array}{l}\text { Equidistant between anterior-posterior } \\
\text { brain }\end{array}$ \\
\hline Saturation pulses & No \\
\hline Slice series & Interleaved \\
\hline Scan time & $2 \mathrm{~min}, 3 \mathrm{sec}$ \\
\hline
\end{tabular}

Gradient echo sequences are the most sensitive sequences for cavernomas. This makes them the preferred sequence for locating cavernomas in patients who tend to have multiple lesions. We will be looking for areas of "blooming" where the lesions appear larger and more indistinct on the gradient echo and $T_{2}$-weighted spin echo images than on the $T_{1}$-weighted spin echo images.

Blooming refers to the increase in signal loss centered on the source that occurs as echo times get longer. For example, a small black dot from a microhemorrhage would grow or "bloom" into a larger black region as the echo time increases.

\section{Sequence 4: Noncontrast coronal multiecho}

18. Run a noncontrast coronal multiecho scan according to Table A2.2.5.

This is a dual echo turbo spin echo sequence that acquires both proton density weighted and $T_{2}$-weighted images simultaneously. On this study, we will be looking for mixed high signal within the center of the lesions, low signal around their periphery, and progressive "blooming" with increased $T_{2}$-weighting between the PD and $T_{2}$-weighted images. The proton density weighted images come as a bonus with the $T_{2}$-weighted images without any additional expenditure of time and may be useful in this situation.

Figure A2.2.1 is the $T_{2}$-weighted image from the noncontrast coronal multiecho scan and beautifully demonstrates the cavernous angioma in the left temporal region.

\section{Sequence 5: Post contrast $T_{1^{-}}$weighted transverse}

Although cavernomas may exhibit enhancement, we will be looking mainly for other enhancement related lesions such as venous angiomas or hemorrhagic tumors. Some of these other lesions may not be appreciated on noncontrast examinations.

Cerebral Venous Lesions

A2.2.5

Supplement 9 


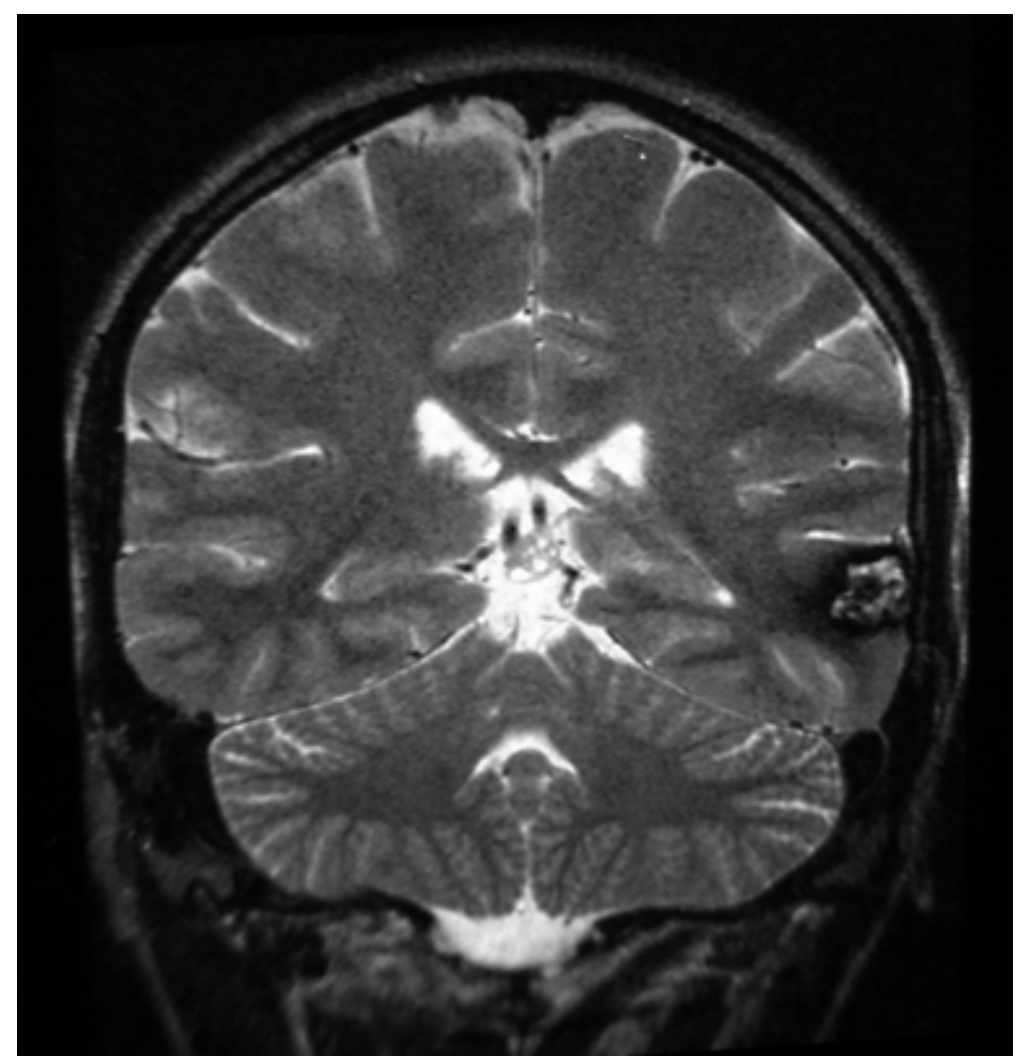

Figure A2.2.1 Coronal $T_{2}$-weighted image.

Table A2.2.6 Imaging Parameters for Sequence 5 (Transverse $T_{1}$-Weighted Spin Echo)

Patient position

Scan type

Imaging plane (orientation)

Central slice or volume center

Echo time $\left(T_{\mathrm{E}}\right)$

Repeat time $\left(T_{\mathrm{R}}\right)$

Flip angle (FA)

Fields of view $\left(\mathrm{FOV}_{\mathrm{x}}, \mathrm{FOV}_{\mathrm{y}}\right)$

Resolution $(\Delta x, \Delta y)$

Number of data points collected $\left(N_{\mathrm{x}}, N_{\mathrm{y}}\right)$

Slice thickness $(\Delta z)$

Number of slices

Slice gap

Number of acquisition $\left(N_{\text {acq }}\right)$

Read direction

Slice location

Saturation pulses

Slice series

Scan time
Supine

Spin echo

Transverse

Slices centered to midbrain on sagittal scout image

$15 \mathrm{msec}$

$560 \mathrm{msec}$

$90^{\circ}$

$230 \mathrm{~mm}, 230 r \mathrm{~mm}$, with $r=3 / 4$ (rectangular field of view)

$0.90 \mathrm{~mm}, 0.90 \mathrm{~mm}$

256, 256r, with $r=3 / 4$ (rectangular field of view)

$6 \mathrm{~mm}$

20

$1.8 \mathrm{~mm}(-30 \%)$

2

Anterior-posterior

Vertex to skull base

None

Interleaved

$3 \mathrm{~min}, 38 \mathrm{sec}$ 
Table A2.2.7 Imaging Parameters for Sequence 6 (Transverse $T_{1}$-Weighted Spin Echo with Flow Compensation)

\begin{tabular}{ll}
\hline Patient position & Supine \\
Scan type & Spin echo \\
Imaging plane (orientation) & Transverse \\
Central slice or volume center & Slices centered to midline on sagittal \\
& scout image \\
Echo time $\left(T_{\mathrm{E}}\right)$ & $17 \mathrm{msec}$ \\
Repeat time $\left(T_{\mathrm{R}}\right)$ & $532 \mathrm{msec}$ \\
Flip angle $(\mathrm{FA})$ & $90^{\circ}$ \\
Fields of view $\left(\mathrm{FOV}, \mathrm{FOV}_{\mathrm{y}}\right)$ & $230 \mathrm{~mm}, 230 \mathrm{rmm}$, with $r=3 / 4$ \\
& $($ rectangular field of view) \\
Resolution $(\Delta x, \Delta y)$ & $0.90 \mathrm{~mm}, 0.90 \mathrm{~mm}$ \\
Number of data points collected $\left(N_{\mathrm{x}}, N_{\mathrm{y}}\right)$ & $256,256 r$, with $r=3 / 4$ (rectangular \\
& field of view $)$ \\
Slice thickness $(\Delta z)$ & $5 \mathrm{~mm}$ \\
Number of slices & 19 \\
Slice gap & $1.5 \mathrm{~mm}(-30 \%)$ \\
Number of acquisitions $\left(N_{\mathrm{acq}}\right)$ & 2 \\
Read direction & Anterior-posterior \\
Slice locations & Vertex to skull base \\
Flow compensation & Yes \\
Saturation pulses & None \\
Slice series & Interleaved \\
Scan time & 3 min, 27 sec \\
\hline
\end{tabular}

19. Remove the patient from the scanner. Do not allow the patient to move on the table. Establish an intravenous line from which the contrast agent can be injected, and attach this line securely to the patient so that movement into or out of the magnet will not pull at the patient's arm. Move the patient back into the scanner.

It is preferable to insert the line prior to imaging and to leave the patient in the magnet, with no intervening motion between the scans run before contrast agent injection and those run after injection.

20. If the patient has moved, run another rapid three-plane positioning pilot scan (see Sequence 1).

21. Leaving the patient in the magnet, inject the contrast agent, flush the line with $10 \mathrm{ml}$ saline, and then immediately run a post contrast $T_{1}$-weighted transverse sequence according to Table A2.2.6.

A dose of $0.1 \mathrm{mmol} / \mathrm{kg}$ of contrast agent is usually given.

Sequence 6: Post contrast $T_{1}$-weighted with flow compensation (optional)

22. In atypical cases where the diagnosis is still in doubt, if indicated to accentuate flow within a lesion, run a post contrast $T_{1}$-weighted sequence with flow compensation according to Table A2.2.7

\section{COMMENTARY}

\section{Background Information}

Cavernomas are usually isolated findings but they may be multiple and familial. They are relatively rare in the general population, but when they occur there tend to be multiple lesions in $\sim 50 \%$ of cases. A familial predilection is seen where up to $80 \%$ of affected family members have multiple lesions. This is particularly seen in the Desert Southwest where there is a strong geographic preponderance for cavernomas. $\mathrm{Pa}$ -
Cerebral Venous Lesions

A2.2.7 
tients of Hispanic extraction more commonly have multiple lesions. Some of these individuals have been linked to a genetic mutation on chromosome seven (Mason et al., 1988).

While cavernomas may occur anywhere in the CNS, $75 \%$ are located in the cerebral hemispheres, with $25 \%$ in the cerebellum or brainstem. When multiple, they may be seen in the spinal cord. Rarely they may be dural, subdural, subarachnoid, or intraventricular. Figures A2.2.2, A2.2.3, and A2.2.4 demonstrate the classic appearance of a cavernoma in an unusual intraventricular location.

A 66-year-old female presented with a history of seizures (Fig. A2.2.2). The scan demonstrates the classic appearance of a cavernoma in an unusual intraventricular location.

Figure A2.2.3 shows the $T_{2}$-weighted image appearance of the same patient as in the previous example. This demonstrates the classic appearance of high central signal surrounded by dark hemosiderin rim.

Figure A2.2.4 shows the unusual intraventricular position of the cavernous angiomas on $T_{1}$-weighted imaging of the same patient as in the two previous examples. While an increasing number of these lesions are found incidentally on routine imaging studies, others will still present with seizures, hemorrhage, focal neurologic deficit, or headache between the ages of thirty and sixty.

Of those presenting with symptoms, $\sim 50 \%$ have had seizures. Frequent occult hemorrhages make the frequency of intracranial hemorrhage difficult to access. While the incidence of subclinical hemorrhage is nearly $100 \%$, the rate of overt hemorrhage leading to clinical symptoms is $\sim 0.5 \%$ to $2 \%$ per year. Approximately $10 \%$ to $15 \%$ of patients with the disease will develop clinically significant hemorrhage (Robinson et al., 1991). The clinical presentation of hemorrhage varies according to location with those in more vital areas such as the brainstem more likely to be symptomatic. Overt hemorrhage is more common in females and is prone to occur during pregnancy.

Grossly the lesions appear multilobulated, like blue-black mulberries, and are more frequent in frontal and temporal lobes. Microscopically, the surrounding brain parenchyma is
Imaging

Cavernous

Malformations

A2.2.8

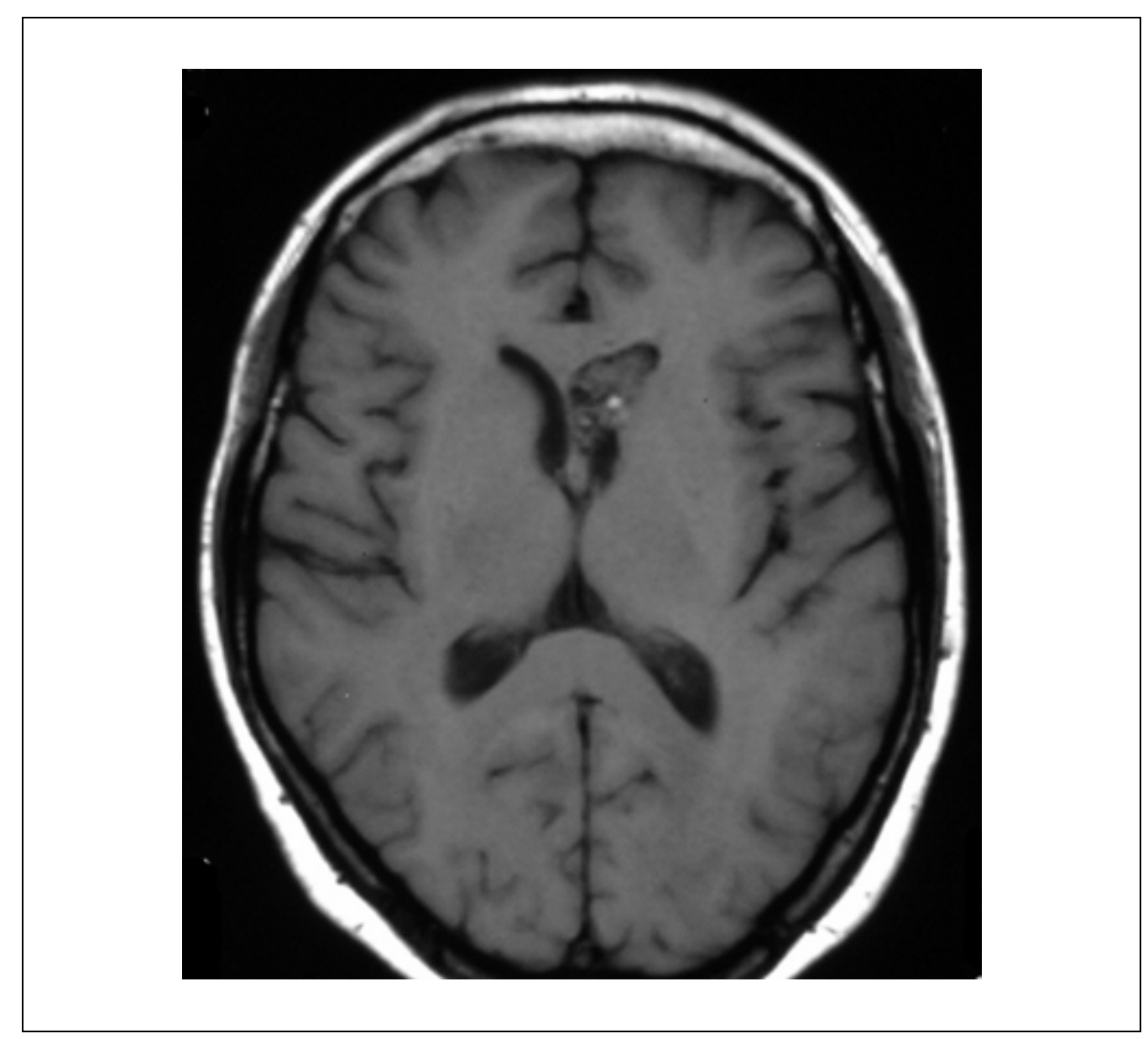

Figure A2.2.2 Transverse $T_{1}$-weighted spin echo image. The classic appearance of a cavernoma in an unusual intraventricular location. 


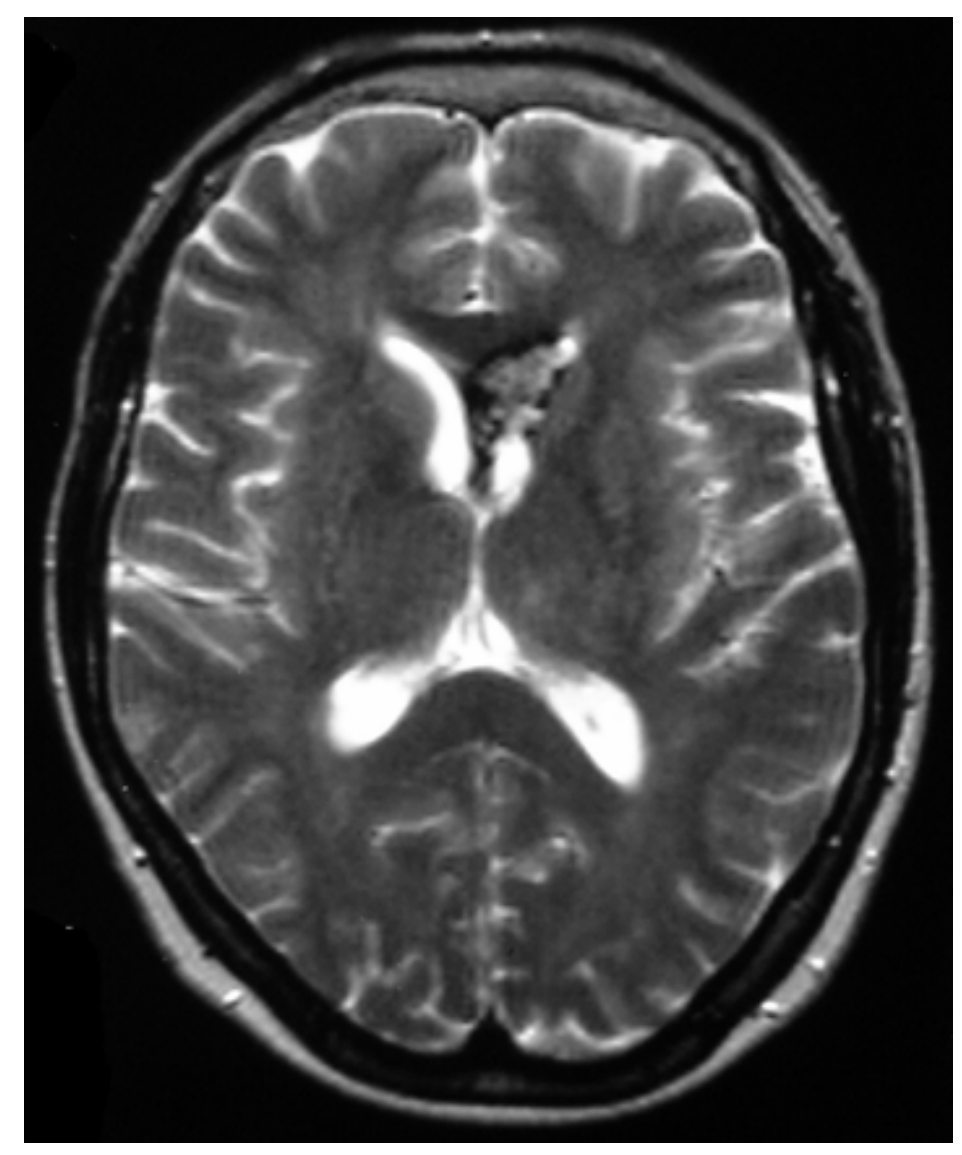

Figure A2.2.3 $\quad T_{2}$-weighted transverse image.

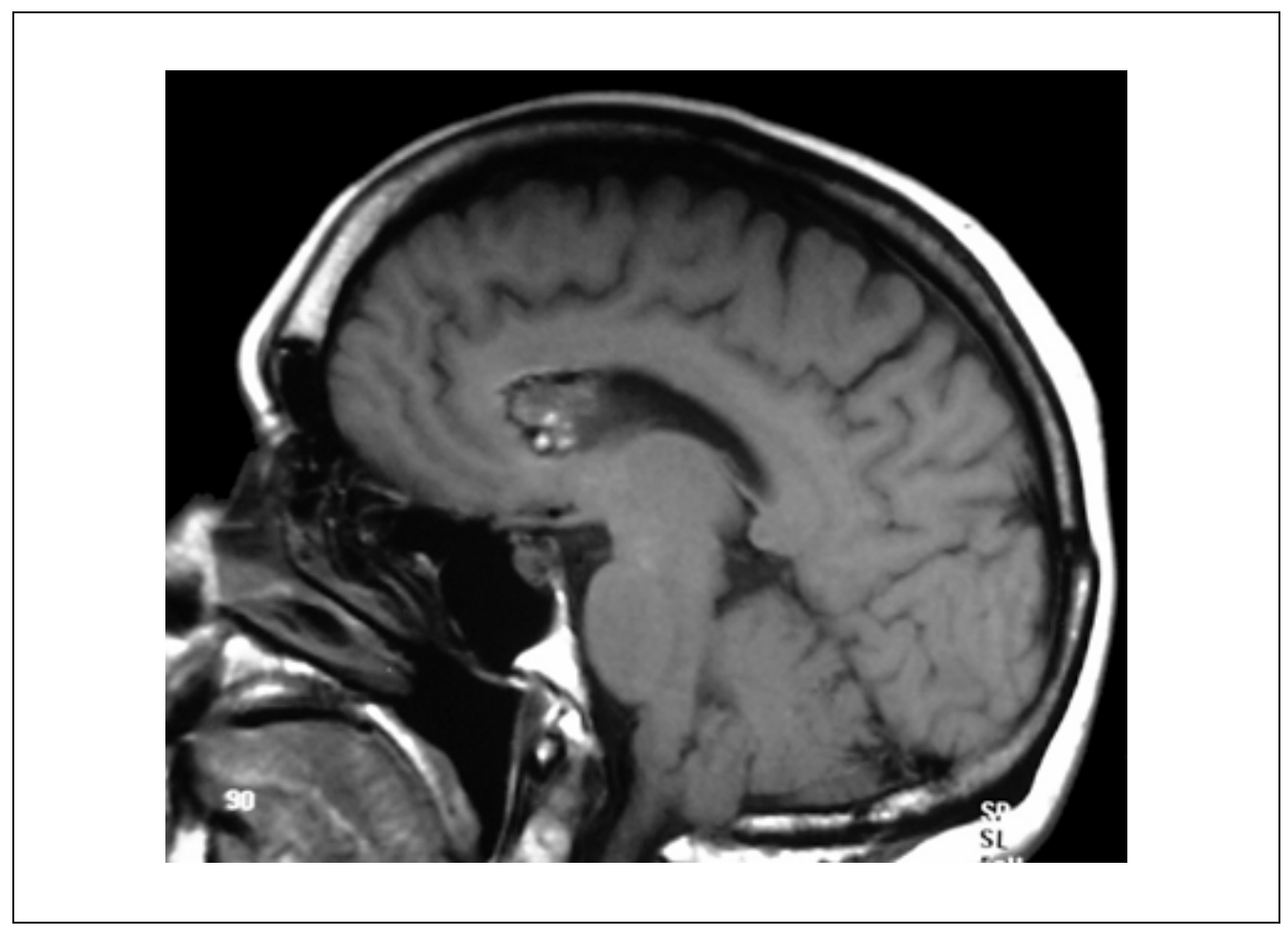

Figure A2.2.4 $\quad T_{1}$-weighted sagittal image without gadolinium. 


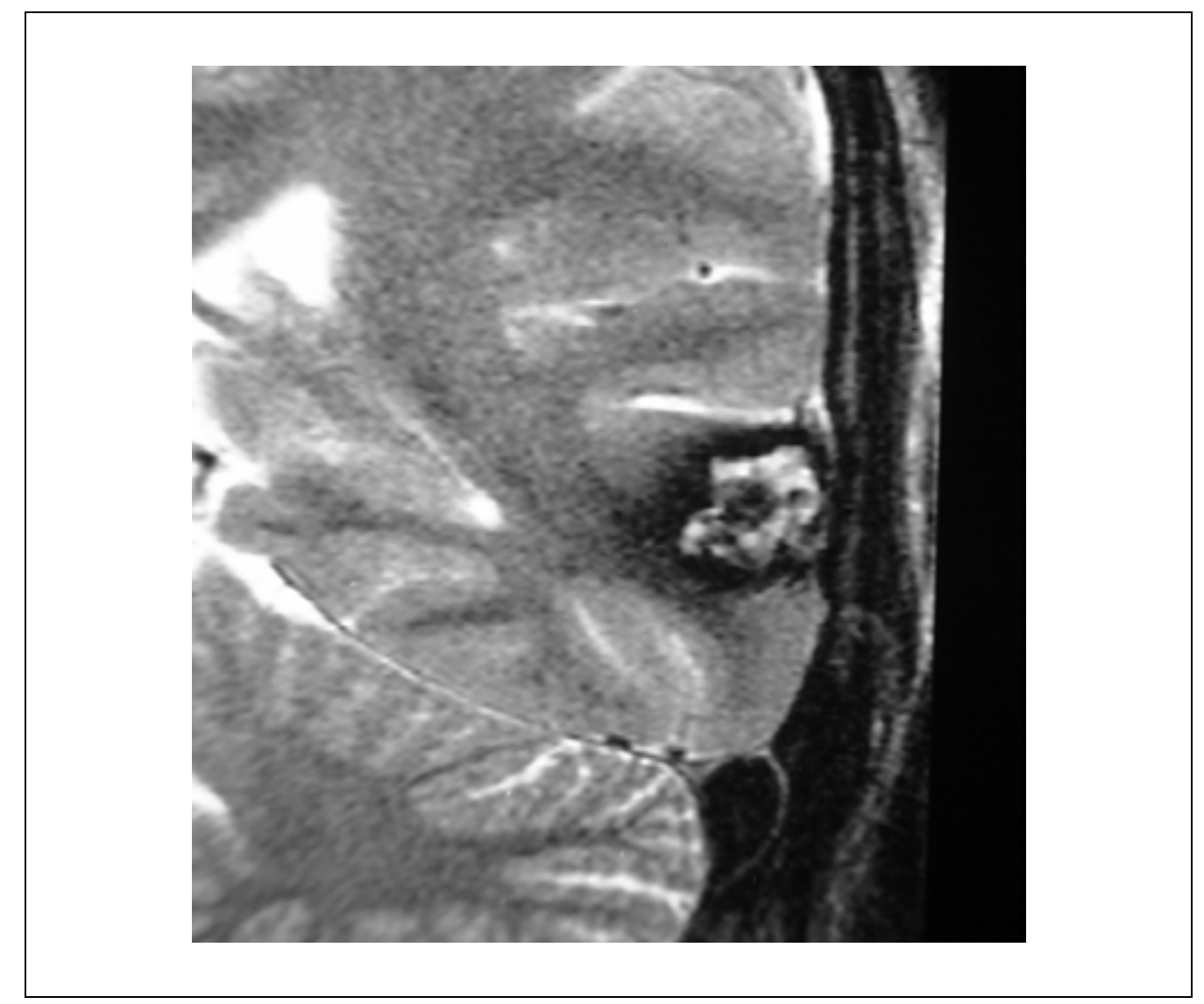

Figure A2.2.5 Noncontrast coronal multiecho $T_{2}$-weighted image.

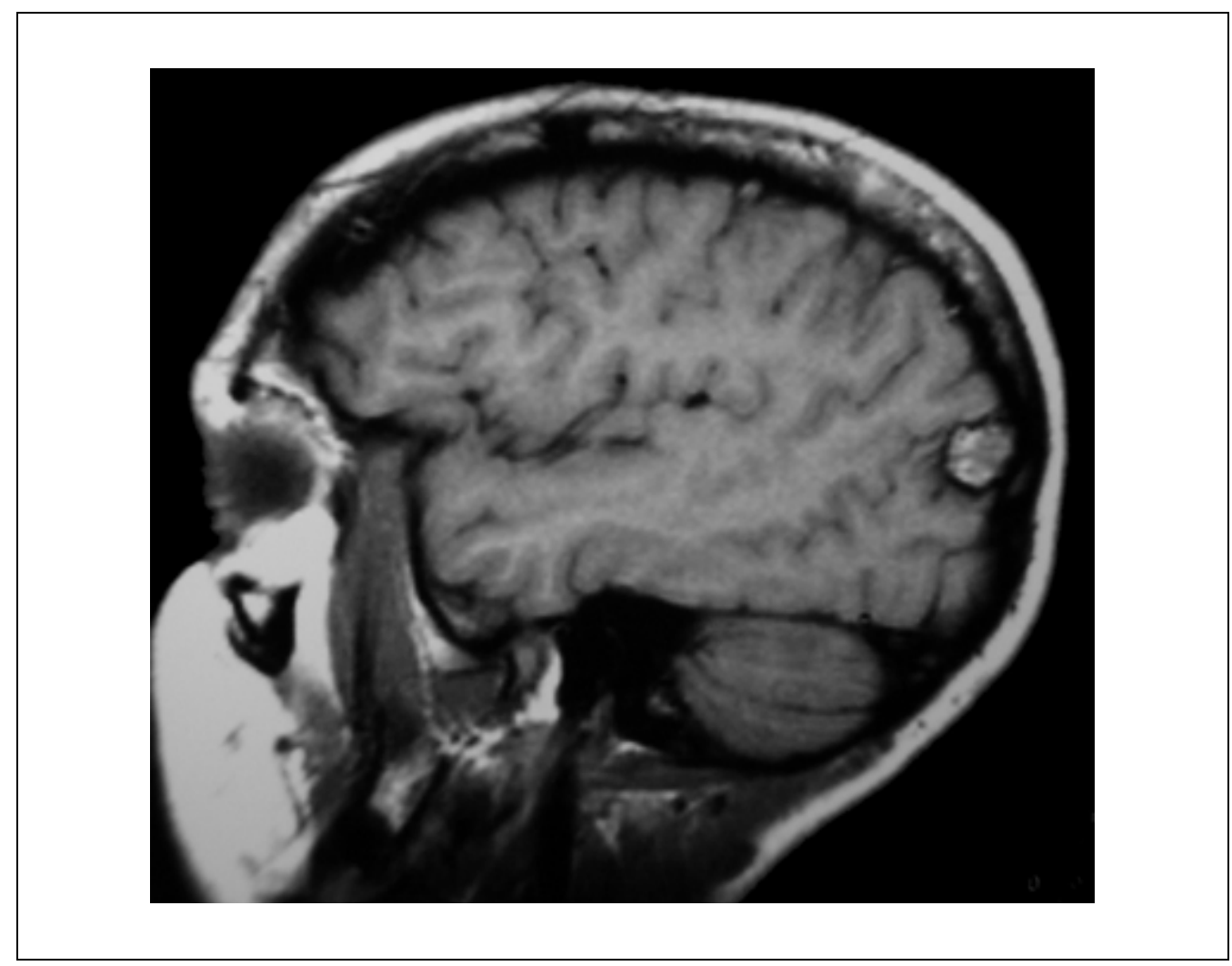

Imaging Cavernous Malformations

Figure A2.2.6 $\quad T_{2}$-weighted sagittal image. 


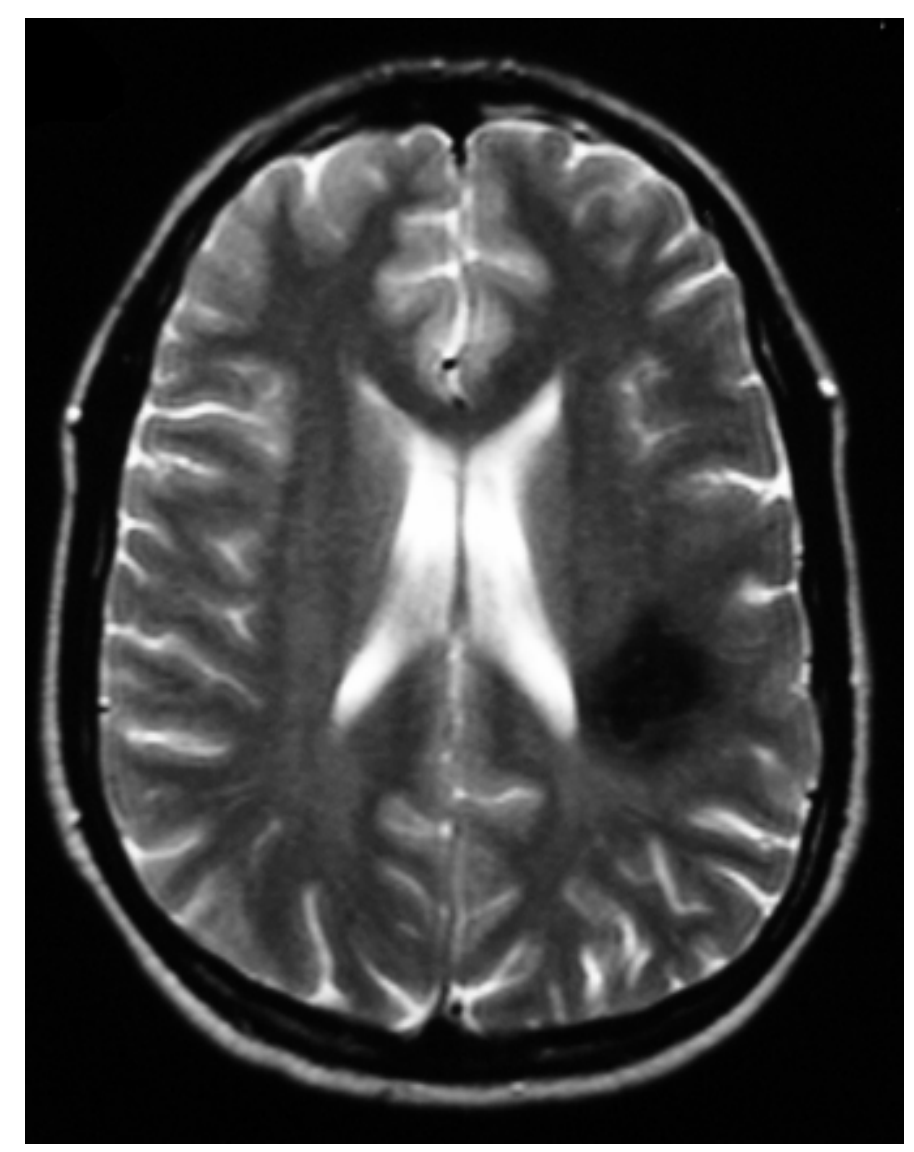

Figure A2.2.7 $\quad T_{2}$-weighted transverse image.

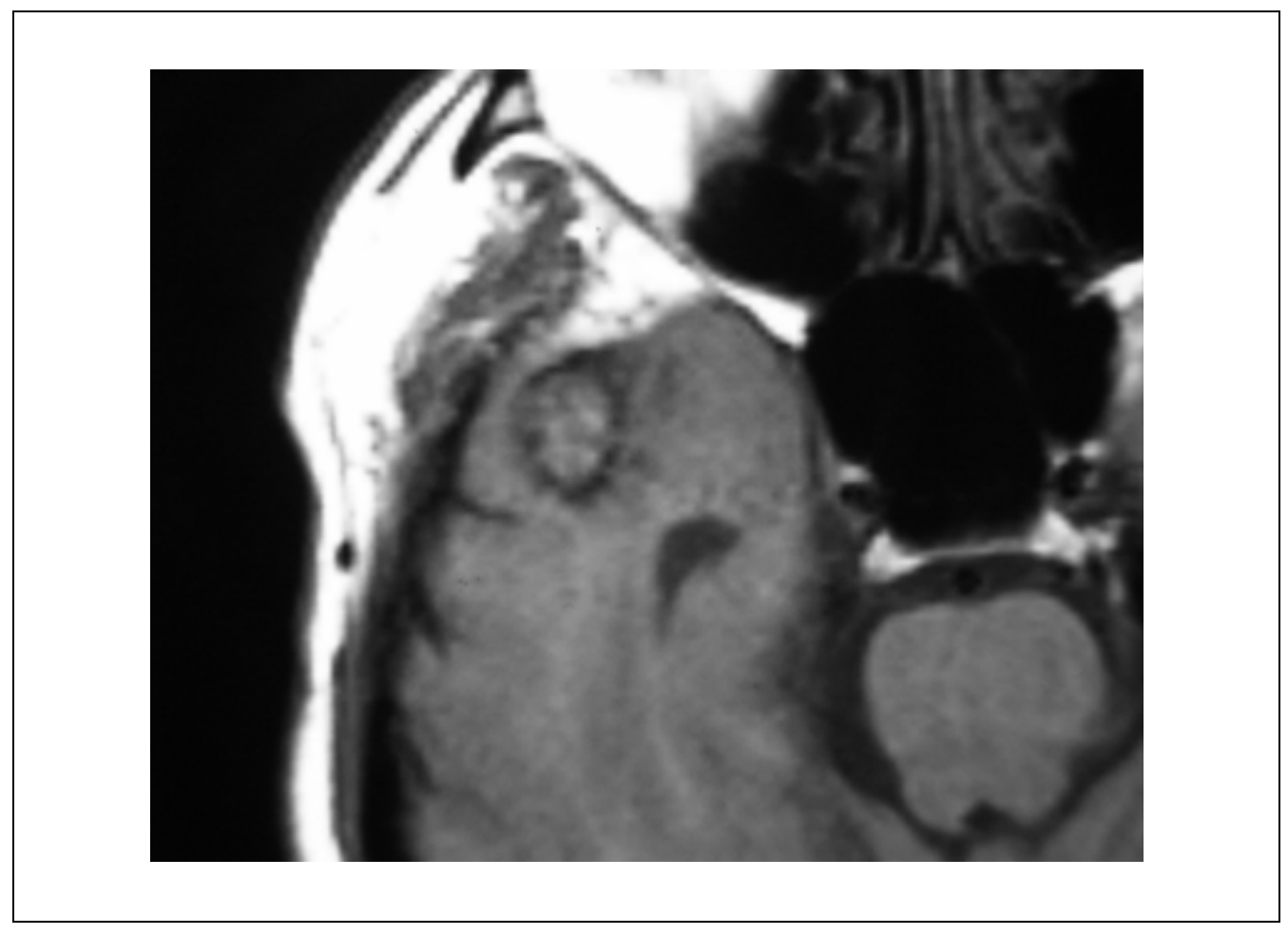

Figure A2.2.8 $\quad T_{1}$-weighted transverse image without gadolinium. 


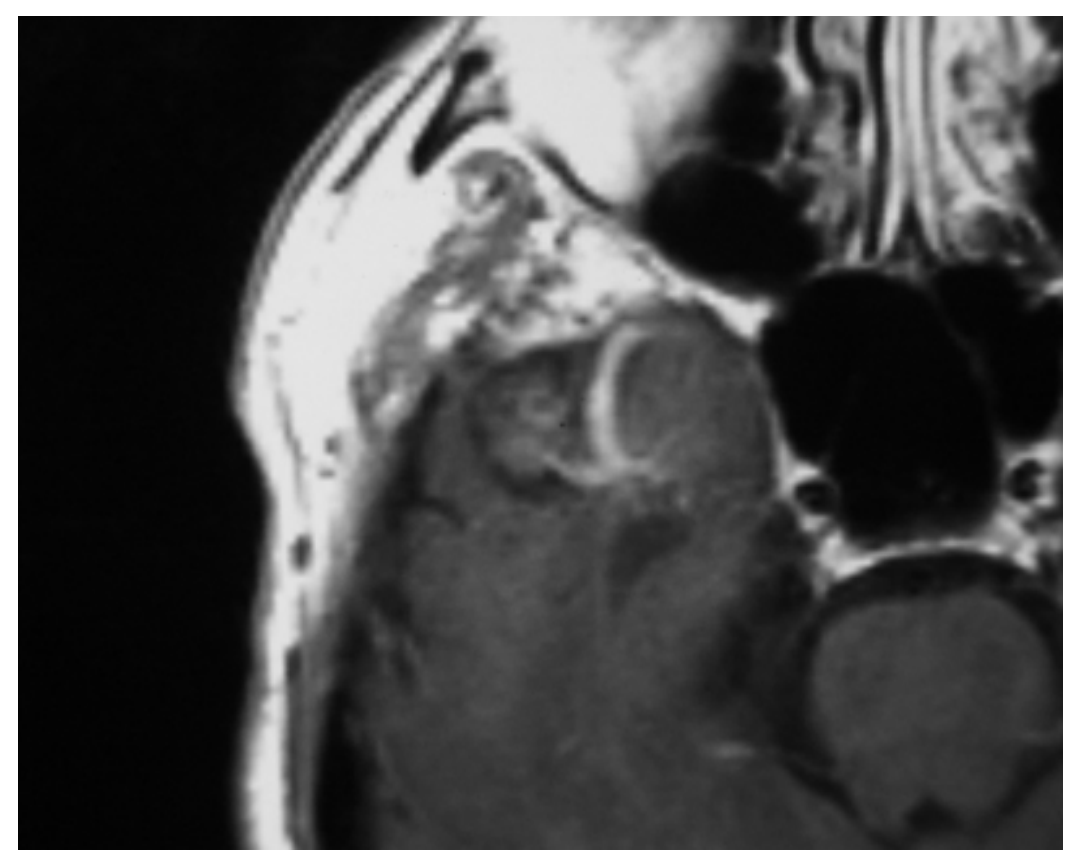

Figure A2.2.9 $\quad T_{1}$-weighted transverse image with gadolinium.

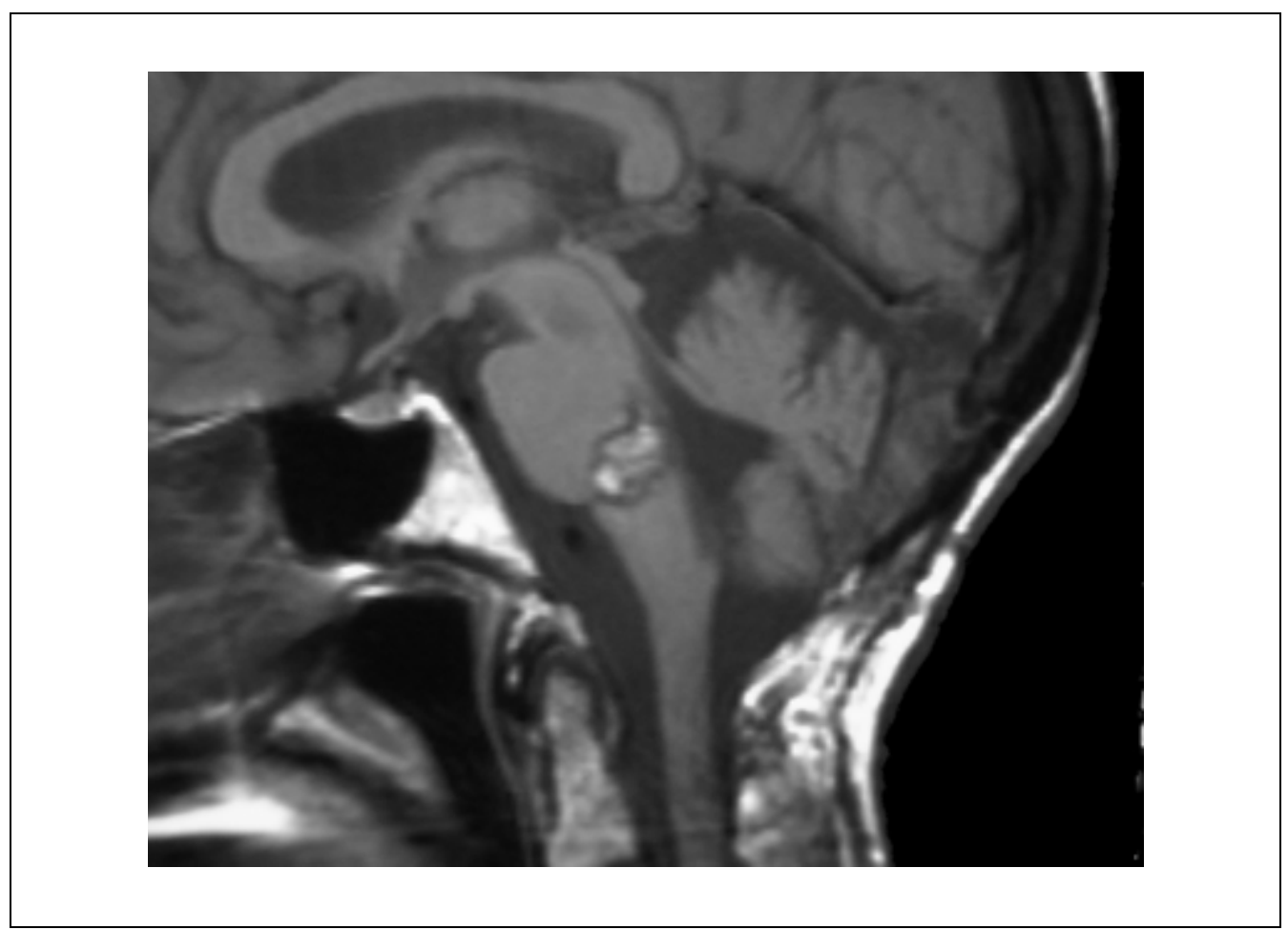

Figure A2.2.10 $T_{1}$-weighted sagittal image without gadolinium.

Imaging Cavernous

Malformations

\section{A2.2.12}




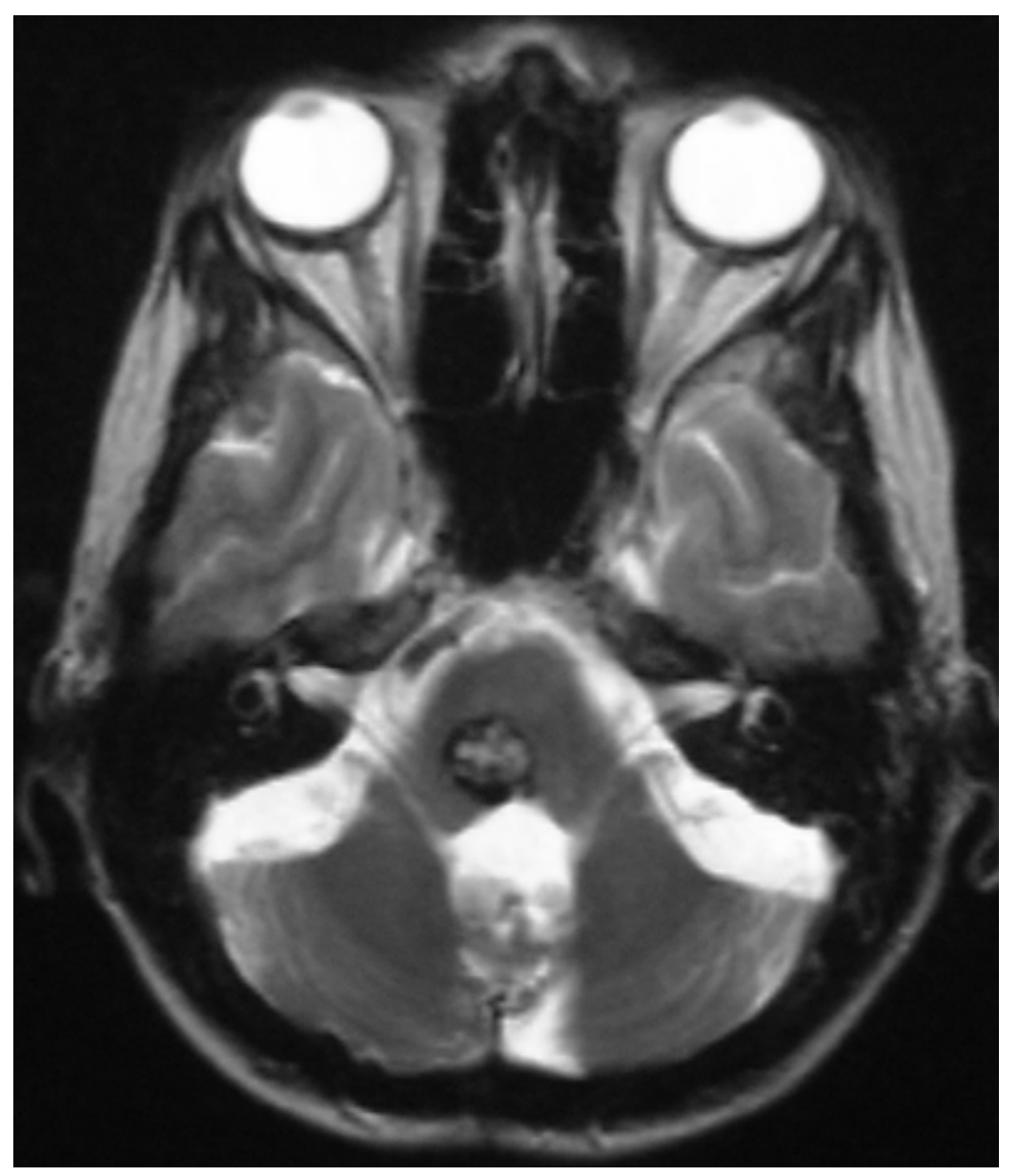

Figure A2.2.11 $T_{2}$-weighted transverse image.

gliotic with hemosiderin staining (Fischbein et al., 2000).

\section{Critical Parameters and Troubleshooting}

Cavernous angiomas often demonstrate susceptibility artifacts that result from incorrect frequency encoding. Heterogeneous susceptibility causes a heterogeneous field. This causes some of the proton's frequency to be mapped incorrectly and result in an inappropriate signal void in the image. A characteristic white band is often seen at the edge of this void. This is due to overlapping signals of protons that are inappropriately mapped. Imagers are familiar with this type of artifact around small bits of metal. Normally we try to minimize susceptibility artifact by using the highest possible read gradient (which often corresponds to the shortest possible $T_{\mathrm{E}}$ in some systems) or by using a fast spin echo technique with the shortest possible spacing (Mitchell, 1999). In this case, we do the opposite, accentuating the artifact and making the lesion more conspicuous. Susceptibility artifact is generally more pronounced with gradient echo techniques. This artifact causes "blooming" and can be seen to be progressively more pronounced with gradient echo sequences and longer $T_{\mathrm{E}}$ 's of $T_{2}$-weighted images.

Figure A2.2.5 is a close up of Figure A2.2.1 and demonstrates indistinctness of the peripheral margin of the hemosiderin ring caused by "blooming." This tends to accentuate the conspicuity of cavernomas with long $T_{\mathrm{E}} T_{2^{-}}$ weighted turbo spin echo and gradient echo sequences.

This increased sensitivity for cavernomas makes gradient echo sequence being the sequence of choice for finding additional lesions that may not be appreciated with conventional imaging techniques. Whereas lesions may be so characteristic that no other lesions are considered, occasionally their appearance may be confusing. Extra-axial lesions typically have high
Cerebral Venous Lesions

A2.2.13 
signal on $T_{2}$-weighted images, more often enhance after gadolinium, and may mimic meningiomas. Intra-axially, classically appearing lesions may turn out to represent hemorrhagic metastasis. In symptomatic patients surgical excision is recommended if feasible with the role of radiosurgery being evaluated (Wilson, 1992).

\section{Anticipated Results}

These lesions usually have a characteristic imaging appearance that is related to the presence of blood products at various evolutionary stages (Rigamonti et al., 1987). The lesion is usually round or lobulated with a mixed signal intensity core.

Figure A2.2.6 shows the $T_{2}$-weighted image appearance in a 28 -year-old male whose lesion was found at a routine MRI examination. This again demonstrates the classic appearance of high central signal surrounded by a dark hemosiderin rim.

There are typically multiple round areas that are bright on both $T_{1}$-weighted and $T_{2}$-weighted images. These rounded deposits cause many of these lesions to have a characteristic "popcorn" appearance. A peripheral ferratin or hemosiderin rim varies in thickness and contour.

Sometimes the hemosiderin rim is so pronounced that only a "black hole" is appreciated on some of the image slices, as is seen on Figure A2.2.7. The adjacent image demonstrated a more classic appearance. The patient is a 38year-old Hispanic female who experienced CNS symptoms each time when she went through one of her pregnancies.

After injection of gadolinium, there may be partial enhancement and an associated venous malformation may be demonstrated.

The next noncontrast $T_{1}$-weighted transverse image (Fig. A2.2.8) demonstrates the cavernous angiomas in the right temporal fossa.

The associated venous angioma was not appreciated until the gadolinium enhanced $T_{1^{-}}$ weighted transverse image (Fig. A2.2.9) was obtained.

Figure A2.2.10 shows the $T_{1}$-weighted image appearance of a 25-year-old female who presented with brainstem symptoms. This again demonstrates the classic appearance in what is not an unusual location.

Figure A2.2.11 shows the $T_{2}$-weighted image appearance of the previous 25-year-old female who presented with brainstem symptoms. The center of the lesion is high signal on both $T_{1^{-}}$ and $T_{2}$-weighted images and there is a dark hemosiderin rim around the periphery of the lesion.

\section{Literature Cited}

Fischbein, N.J., Dillon, W.P., Barkovich, A.J. (eds.) 2000. Teaching Atlas of Brain Imaging, pp. 272275. Thieme, New York.

Glendhill, K., Moore, K.R., Jacobs, M., and Orrison, W.W., Jr. (eds.) 2000. Chapter 21. In Neuroimaging, pp. 252-755. W.B. Saunders, Philadelphia.

Mason, I., Aase, J.M., Orrison, W.W., Wicks, J.D., Seigel, R.S., and Bicknell, J.M. 1988. Familial cavernous Angiomas of the brain in an Hispanic family. Neurology 38:324-326.

Mitchell, D.G. 1999. MRI Principles, pp. 266-267. W.B. Saunders, Philadelphia.

Rigamonti, D., Drayer, B.P., Johnson, P.C., Hadley, M.N., Zabramski, J., and Spetzler, R.F. 1987. The MRI appearance of cavernous malformations (Angiomas). J. Neurosurgery 67:518-524.

Robinson, J.R., Awad, I.A., Little, J.R. 1991 Natural history of the cavernous angioma. J. Neurosurgery 75:709-714.

Shellock, F.G. 1996. Pocket Guide to MR Procedures and Metallic Objects. Lippincott-Raven, Philadelphia.

Tomlinson, F.H., Houser, O.W., Scheithauer, B.W., Sundt, T.M., Jr., Okozaki, H., and Parisa, J.E. 1994. Angiographically occult vascular malformations: A correlative study of features on magnetic resonance imaging and histological examination. Neurosurgery 34:792-800.

Wilson, C.B. 1992 Cryptic vascular malformations. Clinics Neurosurg. 38:49-84.

\section{Key References}

Fischbein, 2000. See above.

These authors afford a current concise look at intracranial vascular malformations. In addition to imaging findings, they discuss etiology, clinical findings, treatment, complications, pearls and pitfalls.

Shellock, 1996. See above.

Covers a number of important patient management issues related to MR imaging, including recommended safety procedures, a list of metallic implants that have been tested for MR compatibility, and a list of other sources on MR safety.

Contributed by F. Allan Midyett

UA North Texas Healthcare

Dallas, Texas

Laurie Fisher

Siemens Uptime Service Center

Cary, North Carolina

Suresh Mukherji

University of Michigan

Ann Arbor, Michigan
Imaging Cavernous Malformations A2.2.14 Supplement 5 